\title{
Characteristics and treatment outcomes of male breast cancer reported to regional cancer centre, India
}

\author{
Pothamsetty RK, Ghosh RR and Thaliath BP \\ Department of Radiation Oncology, Regional Cancer Centre, Kamla Nehru memorial Hospital, Allahabad, UP 211002, India
}

\begin{abstract}
Breast cancer in men is a rare disease and it is not commonly encountered in day to day oncological practice. Male breast cancer accounts for less than $1 \%$ of all cancers in men and less than $1 \%$ of all diagnosed breast cancers [1]. The present study attempted to analyze the clinical profiles and treatment outcomes of breast cancer in men from a regional cancer centre in Eastern Uttar Pradesh, Allahabad, India. The retrospective study was carried out in the Department of Radiation Oncology from January 2013 to January 2015. Data were analyzed in terms of median, standard deviation, frequencies and percentages. Male breast cancer accounted for $0.5 \%$ of all cancer cases diagnosed in males and $2.5 \%$ of all breast cancer cases reported to the hospital during the study period. At the end of last follow up, loco-regional failures, disease free survival, overall survival and distant metastasis analysis showed $4 \%, 43 \%, 74 \%$ and $22 \%$ respectively. Breast cancer do occur in males although its incidence is low, the psychological and social domains to be kept aside and the issue to be addressed seriously. The consensus and guidelines for treating male breast cancer is similar and follow that of female breast cancer till date. The limitations of this study include the retrospective nature of our data and the relatively small number of patients treated over a span of 2 years. More well differentiated research and versatile studies are mandated in this domain to consider male breast cancer as a separate entity or a variant of female breast cancer.
\end{abstract}

\section{Abbrevations}

LVI: Lympho Vascular Invasion; PNI: Peri Neural Invasion; IHC: Immuno Histochemistry; HER 2: Human epidermal growth factoer receptor 2; Ki 67: Profileration Index; TNBC: Triple Negative Breast Cancer; AJCC: American Joint Committee on Cancer; IDC: Infiltrating Ductal Carcinoma; NPI: Nottingham Prognostic Index

\section{Introduction}

Breast cancer in men is a rare disease and it is not commonly encountered in day to day oncological practice. The disease is most commonly seen in elderly population but any age group can be affected. Many of them in the society especially in Indian culture where breast cancer in males are not frequently seen, harbor the notion that breast cancer is a disease of females and males can afford to get any cancer but not breast cancer.

Most of the males especially with breast cancer deferred to visit the doctor owing to the psychological and social aspects. No screening programmes or awareness camps on breast cancer in men were ever being framed or discussed in India as well as in abroad due to its unusual presentation in men. The delay in approaching the physician will significantly transform into advanced stages which significantly alter the prognosis and survival of the patients.

Male breast cancer accounts for less than $1 \%$ of all cancers in men and less than $1 \%$ of all diagnosed breast cancers [1]. Despite, the differences in tumor biological behavior, the stage grouping, metastatic work up and the treatment was almost similar in both male and female breast cancers till date. There were not many cases well documented and the literature was scant emphasizing the male breast cancer.

The present study attempted to analyze the clinical profiles and treatment outcomes of breast cancer in men from a regional cancer centre in Eastern Uttar Pradesh, Allahabad, India.

\section{Objectives}

The study design has set the following objectives-

1. Prevalence of breast cancer in males reported to Regional Cancer Centre, India.

2. Clinical characteristics of male breast cancer.

3. Clinical outcome with respect to loco-regional failures (LRF), disease free survival (DFS) and overall survival (OS).

\section{Research methodology}

The retrospective study was carried out in the Department of Radiation Oncology, Regional Cancer Centre, Kamala Nehru Memorial Hospital, Allahabad, India. All male patients that had histological diagnosis of breast carcinoma from January 2013 to January 2015 were included. We analyzed socio-demographic profile, clinico-pathological entities and survival analysis of the patients. The patients were followed up regularly and for the research purpose, clinical status of the patients were assessed and recorded. The last follow up was done at the end of November 2017. Data were analyzed in terms of median, standard deviation, frequencies and percentages. Detailed case history, tumor biology and socio demographic information was documented. Staging was done based on AJCC 2010. Necessary imaging modalities, tumor biopsies and routine investigations were performed. Additional imaging

Correspondence to: Pothamsetty RK, Department of Radiation Oncology, Regional Cancer Centre, Kamla Nehru memorial Hospital, Allahabad, UP 211002, India, Tel: 919792377423; E-mail: wildfire194@gmail.com

Key words: breast, cancer, male, treatment, outcomes, overall, survival

Received: December 03, 2017; Accepted: December 22, 2017; Published: December 26, 2017 
for metastatic and staging work up as required was investigated as per the individual assessment. Advanced cases were given anthracycline based regimens 4- 6 cycles followed by MRM and irradiation to a dose of 50Gy in 25 fractions in 5 weeks either on Linac Siemens conventional/3DCRT or Cobalt-60. Adjuvant chemotherapy with/ without harmonal therapy was rendered. Harmone positive patients were given tamoxifen. No patient had received trastuzumab in the study group.

\section{Results}

Male breast cancer accounted for $0.5 \%$ of all cancer cases diagnosed in males and $2.5 \%$ of all breast cancer cases reported to the hospital during the study period. The median age at diagnosis was 56 years. The socio-demographic information was recorded and tabulated (Table 1). Right and left breasts were affected in 17 (74\%) and $6(26 \%)$ patients respectively. The most common symptoms were breast lump (52\%), skin ulceration (22\%), nipple discharge (17\%) and others (9\%). The most common location of the tumor was retroareolar (65\%). Histologically, all the patients (100\%) were infiltrating ductal carcinoma (IDC). Most of the patients were presented in stage III $(87 \%)$ of which $39 \%$ in stage IIIB, $26 \%$ in stage IIIC, $22 \%$ in stage IIIA (Figure 1). Tumor pathology depicted most of them were grade II (61\%), and LVI and PNI positivity was seen in 30\% and 9\% respectively (Figures 2 and 3). IHC analysis has revealed that significant number of patients with hormone positivity (56.5\%) and HER2 overamplification was seen in 4\% (Figure 4). Molecular profiling as per IHC, was grouped into 2 groups viz. TNBC and non-TNBC (Figure 5). At the end of last follow up, loco-regional failures, disease free survival and overall survival analysis showed 1 (4\%), $10(43 \%)$ and $17(74 \%)$ patients respectively. Distant metastasis was observed in 5 patients (22\%). Liver (60\%), followed by lungs (40\%) were the sites of metastasis. 1 patient (4\%) during the last follow up, reported to have $2^{\text {nd }}$ primary malignancy in gall bladder, 4 patients (17\%) lost to follow up and 2 patients (9\%) encountered death (Figure 6).

\section{Discussion}

The prevalence of male breast cancer increases with age, and it is rare before the age of 30 years. The most common age group in the study group was 50-59 years (52\%). The median age was 56 years at the time of diagnosis. Most of the studies has concluded that male breast cancer was diagnosed in elderly usually at $6^{\text {th }}-7^{\text {th }}$ decades of life $[2,3]$. In our study, most of the patients presented with right breast cancer unlike other studies which showed predilection for left breast. No bilateral breast carcinoma in the study group and all the patients were exclusively IDC. In the study group, hormone receptor positivity (57\%) is much higher than the female counterparts and is in accordance with other studies [4]. As per the study, TNBC in men is lower compared to the female counterparts. Distant metastasis observed in patients with higher grade and advanced stages.

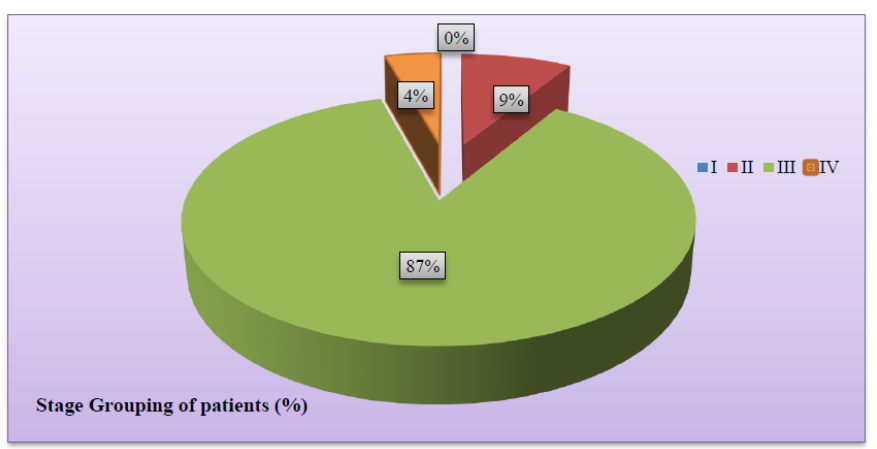

Figure 1. Stage grouping
Table 1. Patient characteristics

\begin{tabular}{|c|c|c|c|}
\hline S. No. & Determinants & No. of Patients & $\%$ \\
\hline 1 & \multicolumn{3}{|c|}{ Socio economic status } \\
\hline & Low- Middle Income & 20 & 87 \\
\hline & High Income & 3 & 13 \\
\hline \multirow[t]{3}{*}{2} & \multicolumn{3}{|c|}{ Demographics } \\
\hline & Rural & 18 & 78 \\
\hline & Urban & 5 & 22 \\
\hline \multirow[t]{5}{*}{3} & \multicolumn{3}{|c|}{ Occupation } \\
\hline & Worker & 7 & 30 \\
\hline & Farmer & 14 & 61 \\
\hline & Business & 2 & 9 \\
\hline & Job holder & 0 & 0 \\
\hline \multirow[t]{3}{*}{4} & \multicolumn{3}{|c|}{ Co-morbidity } \\
\hline & Yes & 6 & 26 \\
\hline & No & 17 & 74 \\
\hline \multirow[t]{3}{*}{5} & \multicolumn{3}{|c|}{ Educational Background } \\
\hline & Literate & 7 & 30 \\
\hline & Illiterate & 16 & 70 \\
\hline \multirow[t]{3}{*}{6} & \multicolumn{3}{|c|}{ Previous Treatment History } \\
\hline & Yes & 4 & 17 \\
\hline & No & 19 & 83 \\
\hline
\end{tabular}

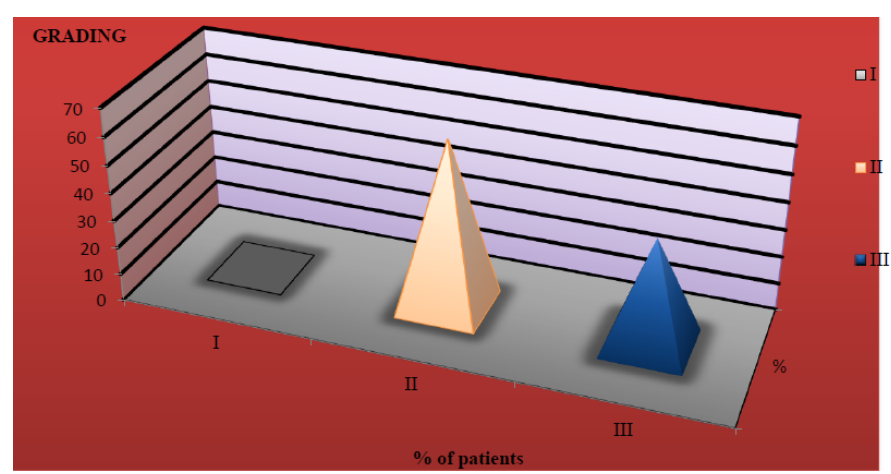

Figure 2. Grading

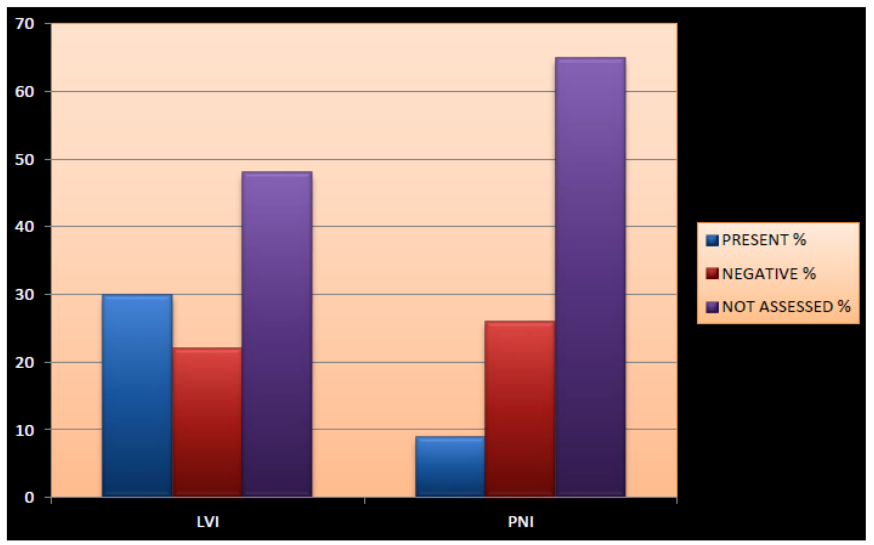

Figure 3. LVI \& PNI

Unlike in females where breast conservation surgeries are widely employed, are not applicable in case of male counterparts. All the patients received MRM and adjuvant loco- regional therapy in the form of radiation has been delivered. All the hormone positive breast cancer patients were received tamoxifen as an adjuvant hormonal therapy. Breast cancer in females is no more a disease of elderly population and the rising trend has been shifted to $<50$ years age group. This is not in 


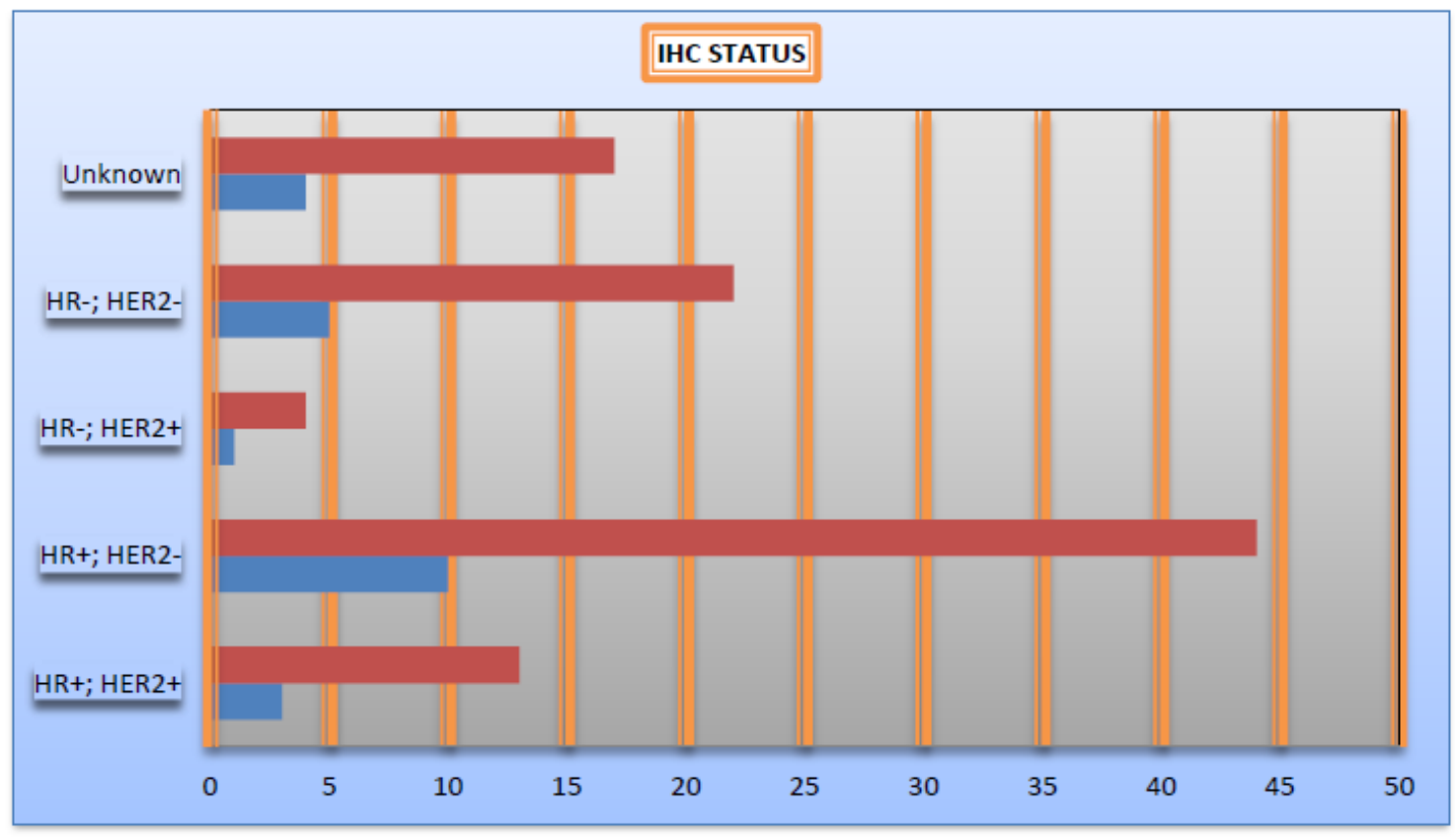

Figure 4. IHC analysis

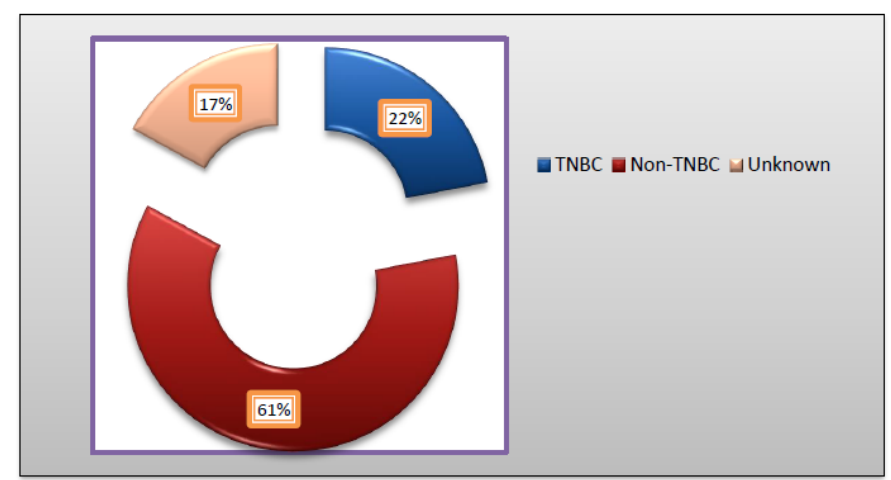

Figure 5. Molecular profiling

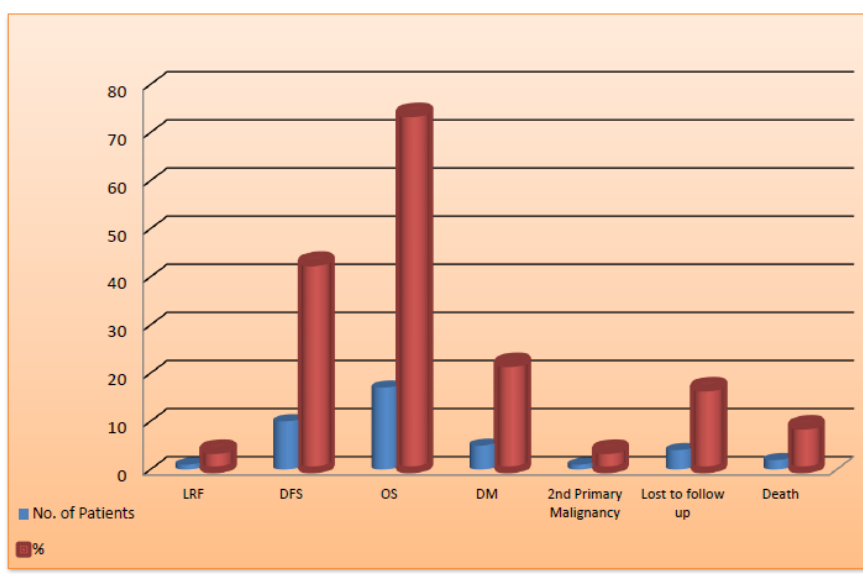

Figure 6. Treatment outcomes

case of males, where advanced cases were seen in elderly population. Male patients as well as the spouse and the family members should discuss the issue, visit the physician and address the issue seriously. The social and cultural aspects of the society should be kept aside in order to manage the issue at appropriate time without any delay for favourable outcome.

The prognostic variables, molecular signatures and genomic sequences which are widely applicable in female breast cancer, can be validated in male counterparts is still a matter of potential debate and investigation. With the evidence been available till now, the treatment of male breast cancer is similar to that of female breast cancer.

\section{Conclusion}

Male breast cancer is a rare disease and occurs in elderly people and most of them presented with advanced stage. Breast cancer in one's life affects multiple dimensions such as financial conditions, public gatherings, psycho-emotional stress, relationship with friends and family, and outlook towards life. Male patients and their family members should discuss the issue and address it seriously keeping aside the psychological and social aspects. Education, camps and oncological conferences or events do play a role in male breast cancer awareness. The treatment of men with breast cancer should be considered at a multidisciplinary forum and approach to management should still follow the established pattern for that of females. The limitations of this study include the retrospective nature of our data and the relatively small number of patients treated over a span of 2 years.

\section{Future implications}

Breast cancer do occur in males although its incidence is low compared to female breast cancer. The consensus and guidelines for treating male breast cancer is similar and follow that of female breast cancer till date. Do the prognostic variables like Ki 67, HER2 over expression or NPI which are being assessed in female breast cancer, can be applied to male breast cancer is still under investigation. Gene signature assays and gene sequencing which are elucidated in female breast cancer can be validated in male breast cancer is still a matter of potential debate and investigation. More well differentiated research and versatile studies are mandated in this domain to consider male breast cancer as a separate entity or a variant of female breast cancer. 


\section{Acknowledgements}

The authors are very grateful to all the consultants whose patients were included in this study. They also appreciate the contribution of all the personnel that participated in the management of these patients.

\section{References}

1. Jemal A, Thomas A, Murray T, Thun M (2002) Cancer statistics 2002. CA Cancer J Clin 52: 23-47.
2. Giordano S, Cohen D, Buzdar AU, Perkins GH, Hortobagyi GN (2003) A populationbased analysis of male breast cancer. J ClinOncol.

3. Fentiman IS, Fourquet A, Hortobagyi GN (2006) Male breast cancer. Lancet 367 595-604.

4. Mitra D, Manna A, Sikdar SK, Sur PK (2007) Clinicopathological study and its prognostic implication in male breast carcinoma. J Indian Med Assoc 105: 681-683, 686. [Crossref]

Copyright: (C)2017 Pothamsetty RK. This is an open-access article distributed under the terms of the Creative Commons Attribution License, which permits unrestricted use, distribution, and reproduction in any medium, provided the original author and source are credited. 